\title{
NADH as a cancer medicine
}

\author{
Michael D. Forrest
}

Department of Computer Science, University of Warwick, Coventry, UK E-Mail: mikeforrest@ hotmail.com

Keywords: NADH, cancer, metabolic disease, cancer metabolism, tumor, tumour, nicotinamide adenine dinucleotide, cure, therapy, drug, Warburg effect, Crabtree effect, aerobic glycolysis, oxidative phosphorylation, OXPHOS, apoptosis, programmed cell death, reactive oxygen species, ROS, oral NADH, NADH supplement, Krebs cycle, TCA cycle, respiration, NAD ${ }^{+}$, ANT 2, lactate, pyruvate, lactate dehydrogenase, dichloroacetate (DCA), frataxin, BMS-199264, $\mathrm{FADH}_{2}$

\begin{abstract}
We propose that NADH will exert a specific kill action against some cancers. NADH is a natural metabolite. We envisage a low side effect profile and that NADH therapy will, additionally, combat the wastage and weakness of cancer patients, which can be the cause of death in some cases. Significantly, NADH can be administered orally and has already cleared clinical trials, all be it for other pathologies.
\end{abstract}

\section{Background}

Aerobic respiration consists of glycolysis in the cytoplasm and in the mitochondria: the Krebs cycle and oxidative phosphorylation (OXPHOS) [1,2]. It requires $\mathrm{O}_{2}$ and net yields 30 ATPs from one glucose molecule. Anaerobic respiration, consisting of glycolysis only, does not require $\mathrm{O}_{2}$ but produces merely 2 ATPs from one glucose molecule. When $\mathrm{O}_{2}$ is available, normal animal cells tend to favour aerobic respiration because of its higher ATP yield.

Aerobic glycolysis is the sole use of glycolysis to produce ATP, even in the presence of $\mathrm{O}_{2}$. Pyruvate and NADH, the outputs of glycolysis, are not fed into the further, mitochondrial steps of the Krebs cycle and OXPHOS to gain a higher ATP yield; despite the presence of $\mathrm{O}_{2}$. Pyruvate is converted to lactate, which requires all the glycolytic NADH output to be converted to $\mathrm{NAD}^{+}$, and this lactate is then excreted from the cell. So, aerobic glycolysis does not net output any NADH.

Cancer cells use aerobic glycolysis some or all of the time 
Cancer cells produce ATP through aerobic glycolysis (Warburg effect) some or all of the time [3-7]. This metabolic fingerprint is already exploited clinically to diagnose cancer using the radiolabeled glucose analogue $-{ }^{18}$ Fluoro-deoxyglucose (FDG) - in positron emission tomography (PET) [8]. Cancer cells utilising aerobic glycolysis have higher glucose uptake rates than surrounding, untransformed cells utilising aerobic respiration. It is this disparity that permits tumour identification. The vast majority of metastatic tumours are highly glycolytic (>90\%) [9-10] and it may be that all of them are, but not all can be resolved at the present sensitivity and selectivity of the technique [11] e.g. those smaller than $0.8 \mathrm{~cm}^{3}$.

Warburg suggested that cancer cells use aerobic glycolysis because their OXPHOS apparatus is injured, and that this damage is a necessary step on the progression to a metastatic phenotype [3]. This is not always true. Yes, some cancer cell lines use aerobic glycolysis always and some do have defective OXPHOS apparatus. However, others use it only for a proportion of the time; using aerobic respiration at other times [6, 12-14]. They can switch between glycolytic and oxidative metabolism in a reversible fashion. For example, the AS30D rat hepatoma cell line uses aerobic glycolysis for $60 \%$ of the time and aerobic respiration for the remainder [15]. A tumour of this cell line should still always be identifiable by PET because the cancerous cells are not all in metabolic sync - at any one time, a proportion of the cells will always be expressing aerobic glycolysis even if others are utilising aerobic respiration. Some cancer cells can utilise OXPHOS but exhibit a glucose-induced switch into aerobic glycolysis (Crabtree effect [16]).

\section{Why do cancer cells employ aerobic glycolysis?}

Why do cancer cells utilise aerobic glycolysis some or all of the time? I propose that they need to use aerobic glycolysis, at the very minimum, during some crucial points in their proliferation cycle; for example, during DNA replication (S phase). This is a deduction from the fact that aerobic glycolysis is not restricted to cancer cells, but is applied by other highly proliferating cells e.g. embryonic cells [17-18]. For example, proliferating thymocytes switch from oxidative to glycolytic metabolism [19-24] during the $G_{1} / S$ transition of their cell cycle [19]. Their increasing dependence upon glycolysis peaks in concert with DNA replication [20]. OXPHOS produces harmful Reactive Oxygen Species (ROS), in addition to useful ATP, and these can damage cellular componentry. I propose that this is a tolerated evil normally but when cells are proliferating at an appreciable rate, and significant DNA replication is occurring, cells deliberately shunt OXPHOS. They do this by switching into aerobic glycolysis, which doesn't produce or result in any NADH or FADH $\mathrm{F}_{2}$ substrate for OXPHOS. In support, proliferating thymocytes employ aerobic glycolysis and produce minimal ROS in contrast to resting thymocytes, which use OXPHOS [24]. DNA replication is a vulnerable stage because DNA is unwound and exposed (from protective chromatin/histones [1-2]); in addition any damage at this stage could find itself reliably 
propagated into daughter cells, and further progeny downstream, before it can be identified as damage and repaired.

This peculiar metabolism may have an additional, insidious benefit for cancer cells. ROS are pivotal to apoptotic pathways $[1,2]$. So with little or none produced, the cancer cell may then be liberated from the apoptotic contingency and free to conduct itself pathologically. Furthermore, although apoptosis is destructive, it is an active process and requires energy expenditure. By shutting mitochondria out of ATP generation it delimits their energy supply and may constrain their ability to initiate and pursue apoptosis. Furthermore, during aerobic glycolysis, the glycolytic enzyme hexokinase binds the voltage-dependent anion channel (VDAC) in the mitochondrial membrane and this suppresses apoptosis [25-26].

Aerobic glycolysis may also permit cancer cells to thrive in the hypoxia of solid tumours [27]. Furthermore, although it conveys a lower ATP yield than OXPHOS, it can produce ATP much faster $(* 100)$ [28] and so can in fact deliver more ATP than OXPHOS, if the substrate supply isn't limiting. Cancer cells fast use of substrate denies it to surrounding untransformed cells and conveys a selective advantage [28]. Furthermore, it may produce local acidosis which may destroy adjacent, normal populations [11]. Greater glycolysis is good for proliferation because glycolytic intermediates are needed for macromolecular biosynthesis $[17,28]$. They are needed for the pentose phosphate pathway that produces $\mathrm{NADPH}$, which is needed for glutathione-dependent anti-oxidant mechanisms [29].

Cancer cells using aerobic glycolysis excrete lactate which may be then taken up and oxidised by other cancer cells using oxidative metabolism [30]. A given cancer cell may switch between the export and import of lactate, depending on where it is at in its proliferative cycle and whether it is employing aerobic glycolysis or oxidation. So, as a system, the tumour may in fact be gaining a full ATP yield from glucose. Incidentally, lactate transports (MCT $1 \& 4$ ) are an excellent drug target; although they will presumably require that the patient forgoes anaerobic exercise during treatment. An alarming heart rate monitor could be used to manage this constraint.

\section{If a cancer cell is switched out of aerobic glycolysis, into using OXPHOS, it can kill the cancer cell}

As aforementioned, a cancer cell may use aerobic respiration for a significant proportion of its time. But I propose that at key times in the proliferating cycle it must instead be using aerobic glycolysis and if it isn't, it will be driven into apoptosis. There is experimental evidence for this. 
Pyruvate is the end product of glycolysis. In aerobic glycolysis, pyruvate is converted to lactate and excreted. Lactate dehydrogenase (LDH) catalyses this reaction. In aerobic respiration, pyruvate dehydrogenase (PDH) out-competes LDH for pyruvate and switches it into the Krebs cycle. During aerobic glycolysis, PDH is inhibited by pyruvate dehydrogenase kinase (PDK), which can itself be inhibited by the dichloroacetate (DCA) drug [31]. So, the DCA drug can switch a cell from aerobic glycolysis to oxidative respiration, with its constituent Krebs and OXPHOS components. This drug induced conversion switches cancer cells into apoptosis [31]. Indeed, DCA in the drinking water of rats can prevent and reverse tumour growth in vivo. DCA is an exciting cancer drug prospect and is already in clinical use for other applications. OXPHOS producing ROS is the trigger for apoptosis in these cancer cells [31].

Why should OXPHOS producing ROS be doomsday for cancer cells but permissible for nontransformed cells? I propose that it relates to proliferation. When cells are proliferating and DNA is unwound, unprotected and being replicated: if high ROS levels are sensed at this time, the process is aborted and the cell is driven into apoptosis. At other times, outside of this key stage(s) in the proliferation cycle, ROS are tolerated and the cell may utilise OXPHOS. The duration of this sensitive period may be quite narrow. I suggest that if one makes a proliferating cell constitutively use OXPHOS, robbing it of the ability to switch off OXPHOS at key stages, one will push it into apoptosis. Or at the very least, halt its proliferation. There is experimental evidence supporting the former [31] and the latter [23]. The latter study relates to healthy proliferating cells, rather than cancer cells. It shows that these issues of aerobic glycolysis are wider than cancer cells and may encompass all highly proliferating cells.

In conclusion, I propose that the use of aerobic glycolysis by cancer cells is not a function of their pathology but is a normal, physiological state employed by highly proliferating cells. If they are not in this state during key stages of the proliferating process, there are other physiological mechanisms that will halt their proliferation or drive them into apoptosis. This is a safety measure to ensure that ROS don't damage DNA during the DNA replication process. DNA damage cannot be afforded at this stage because there isn't the time for it to be repaired before the DNA strands are separated, and with this separation the record of damage is lost forever and the damage is rendered into permanence.

\section{Further evidence}

Increasing the $\mathrm{NAD}^{+}$level, by a treatment with its pre-cursers, is observed to have an anticancer effect [32]. The last step of aerobic glycolysis produces lactate and $\mathrm{NAD}^{+}$. I propose that with an imposed, increased $\mathrm{NAD}^{+}$level, this reaction is more unfavourable. Especially as 
compared to the competing reaction: so pyruvate and NADH are more liable to enter the Krebs cycle and OXPHOS respectively. ROS are then produced that halt proliferation and trigger apoptosis. This rationale also explains why [32] found increased Complex I activity to be unconducive to cancer proliferation and survival.

Friedreich ataxia is an inherited neurodegenerative disorder caused by a reduced expression of frataxin, which results in less OXPHOS and ATP synthesis. Overexpressed frataxin stimulates OXPHOS, and ATP production, in colon cancer cells [33]. This stimulation suppresses colony formation in vitro and tumour formation/growth in nude mice; "these results support the view that an increase in oxidative metabolism (induced by mitochondrial frataxin) may inhibit cancer growth in mammals" [33].

\section{NADH as a cancer cure}

I propose that exogenous NADH administration will cure some cancers. NADH is a substrate for OXPHOS and a continuous supply will confer a constitutive, enduring utilisation of OXPHOS in cancer cells. This will either halt their proliferation or trigger apoptosis.

Exogenous NADH will be harmless to most normal cells. It will just provide an additional source of energy for them. They will lower their glycolytic and TCA cycle rate accordingly to produce less NADH themselves (via ATP's allosteric feedback mechanisms upon key glycolytic enzymes [1,2]). Exogenous NADH may, however, be harmful to non-malignant, proliferating cells. So, there could be side effects. Although, in an adult, the proportion of healthy cells that proliferate at high rates is very small. So, these are likely to be slight.

The anti-cancer effect of a number of conventional treatments is based on their ability to stimulate ROS production and then apoptosis [34] e.g. radiation, etoposide, arsenates, vinblastine, cisplatin, mitomycin $\mathrm{C}$, doxorubicin, camptothecin, inostamycin, neocarzinostatin etc. These typically stimulate ROS production, and damage, across all cells: cancerous or healthy. Cancer cells may be affected more because proliferating cells have a lower tolerance for ROS before apoptosis is initiated, as discussed. But normal cells are also damaged and the side effect profiles can be horrendous. Contrast this with the more targeted action of NADH therapy.

\section{NADH delivery}

How can NADH be delivered into cells? NADH transports can be expressed at the surface of eukaryotes [35] and prokaryotes [36]; extracellular NADH can reach the OXPHOS apparatus 
and increase intracellular ATP [37]. NADH can be successfully administered orally or by intravenous/intraperitoneal infusion [38].

\section{NADH may combat the weight loss, wastage and weakness of cancer patients}

Exogenous NADH may help the energy state of tumour bearing organisms, which typically suffer severe weight loss, wastage and weakness [39]. This is a seriously debilitating and upsetting aspect of cancer for patients. It can even be the cause of death. Wastage may happen because of the inefficiencies of cancer metabolism and the requirement to recycle glucose from lactate in the liver. This requires 6 ATP per lactate molecule (Cori cycle, [1-2]) whereas a molecular conversion of glucose to lactate in cancer cells only yields 2 ATP.

ATP infusions have been trialled to remedy this wastage of cancer patients, with some success [40-47]. However, it is unclear how ATP can enter cells. It is widely believed that mammalian cells cannot import external ATP (although this has been disputed, [47]). NADH therapy may produce better results as there is thought to be NADH transports at mammalian cellular surfaces [35, 37] and NADH can be given orally [38] rather than by long - and so by necessity, infrequent - periods of intravenous infusion.

\section{NADH will not work for cancer cell lines that have a defective OXPHOS apparatus}

Some cancer cells have a working OXPHOS apparatus and some may use it some of the time. Others have a defective OXPHOS apparatus and are wholly reliant on aerobic glycolysis. These latter lines will be resistant to NADH treatment. However, their complete reliance on glycolysis will make them completely fallible to drugs that inhibit aerobic glycolysis [49-55] or glycolysis more generally. Although - note - drugs targeting generic glycolysis can cause severe side effects [56]. NADH therapy should be used first and if this fails then it can be surmised that the cancer cannot respire aerobically and is fully reliant upon glycolysis. Thus, its vulnerability is found. NADH therapy - with its (anticipated) low side effect profile - can be a curing step and, in failure, a screening step for lines susceptible to glycolytic inhibitors. Alternatively NADH therapy can be administered alongside a ketogenic diet (low glucose/carbohydrate, high fat), which may "starve" cells relying singly on glycolysis [57]. $\mathrm{NADH}$, as a natural metabolite, may be permissible to communities and religions that prohibit the use of drugs, even in life threatening cases.

\section{$\mathrm{FADH}_{2}$ therapy}

Exogenous $\mathrm{FADH}_{2}$ could be used as an alternative to $\mathrm{NADH}$, which would circumnavigate Complex I and could be advantageous to destroying cancer cells that have developed a 
defective form of it. Although we would first need to discover whether exogenous $\mathrm{FADH}_{2}$ can passage the plasma and mitochondrial membranes.

\section{Prior work with NADH}

NADH has been reported to have a kill effect upon some cancer cell lines in vitro [58] and in human case studies, where it was orally administered [59]. Unfortunately, the latter are anecdotal and lack rigor; further work is needed. The in vitro studies show, as anticipated, that NADH cannot kill all cancer cell lines. As aforementioned, I propose that those with a defective OXPHOS apparatus are immune to it.

\section{Conclusion}

I propose that NADH pills will cure some cancers. A natural metabolite exerting such a specific kill effect is counterintuitive and intriguing. NADH therapy may also combat the wastage and weakness of cancer patients. Crucially, oral NADH has been proven as safe for humans in clinical trials (as a treatment for depression, Parkinson's disease and chronic fatigue syndrome [60-63]).

This paper presents theory rather than data; but it is theory that is desperately needed at this point. Drug discovery should be driven by rationale rather than a random walk through compounds, awaiting fortuity and serendipity. We must rethink our approaches. We haven't significantly improved the survival of Stage IV cancer patients since President Richard M. Nixon declared war on cancer over 40 years ago. My hope is that this article will prompt investigators to pursue directed animal and clinical studies.

\section{REFERENCES}

[1] Alberts B, Johnson A, Lewis J, Raff M, Roberts K, Walter P (1994) Molecular Biology Of The Cell, $3^{\text {rd }}$ Ed. New York, NY: Garland Publishing.

[2] Stryer L, Berg JM, Tymoczko JL (2002) Biochemistry, $4^{\text {th }}$ Ed. New York, NY: WH Freeman.

[3] Warburg O (1956) On the origin of cancer cells. Science 123(3191):309-314.

[4] Ferreira LM (2010) Cancer metabolism: the Warburg effect today. Exp Mol Pathol 89(3):372-380.

[5] Kim JW, Dang CV (2006) Cancer's molecular sweet tooth and the Warburg effect. Cancer Res 66(18):8927-8930. 
[6] Lopez-Lazaro M (2008) The warburg effect: why and how do cancer cells activate glycolysis in the presence of oxygen? Anti-Cancer Agent Me 8(3):305-312.

[7] Elliott RL, Jiang XP, Head JF (2014) Want to Cure Cancer? Then Revisit the Past; "Warburg Was Correct", Cancer Is a Metabolic Disease. Journal of Cancer Therapy

[8] Bomanji JB, Costa DC, Ell PJ (2001) Clinical role of positron emission tomography in oncology. Lancet Oncol 2(3):157-164.

[9] Czernin J, Phelps ME (2002) Positron emission tomography scanning: current and future applications. Annu Rev Med 53:89 - 112.

[10] Gambhir SS, Czernin J, Schwimmer J, Silverman DH, Coleman RE, Phelps MEA (2001) Tabulated summary of the FDG PET literature. J Nucl Med 42:1S-93S.

[11] Gatenby RA, Gillies RJ (2004) Why do cancers have high aerobic glycolysis? Nat Rev Cancer 4(11):891-899.

[12] Birsoy K, Possemato R, Lorbeer FK, Bayraktar EC, Thiru P et al. (2014) Metabolic determinants of cancer cell sensitivity to glucose limitation and biguanides. Nature 508(7494):108-112.

[13] Fantin VR, St-Pierre J, Leder P (2006) Attenuation of LDH-A expression uncovers a link between glycolysis, mitochondrial physiology, and tumor maintenance. Cancer cell 9(6):425-434.

[14] Bui T, Thompson CB (2006) Cancer's sweet tooth. Cancer cell 9(6):419-420.

[15] Nakashima RA, Paggi MG, Pedersen PL (1984) Contributions of glycolysis and oxidative phosphorylation to adenosine 5 '-triphosphate production in AS-30D hepatoma cells. Cancer Res 44(12 Part 1):5702-5706

[16] Diaz-Ruiz R, Rigoulet M, Devin A (2011) The Warburg and Crabtree effects: On the origin of cancer cell energy metabolism and of yeast glucose repression. BBA-Bioenergetics 1807(6):568-576.

[17] Lunt SY, Vander Heiden MG (2011) Aerobic glycolysis: meeting the metabolic requirements of cell proliferation. Annu Rev Cell Dev Bi 27:441-464.

[18] Kondoh H, Gil J (2006) Protection from the senescence effects of oxidative stress in ES cells. Stem Cell Therapy. Nova Publishers, USA.

[19] Greiner EF, Guppy M, Brand K (1994) Glucose is essential for proliferation and the glycolytic enzyme induction that provokes a transition to glycolytic energy production. J Biol Chem 269(50):31484-31490.

[20] Brand K (1985) Glutamine and glucose metabolism during thymocyte proliferation. Biochem J 228:353-361. 
[21] Brand KA, Hermfisse U (1997) Aerobic glycolysis by proliferating cells: a protective strategy against reactive oxygen species. FASEB J 11(5):388-395.

[22] Brand K (1997) Aerobic glycolysis by proliferating cells: protection against oxidative stress at the expense of energy yield. J Bioenerg Biomembr 29:355-364.

[23] Aulwurm UR, Brand KA (2000) Increased formation of reactive oxygen species due to glucose depletion in primary cultures of rat thymocytes inhibits proliferation. Eur J Biochem 267:5693-8.

[24] Brand K, Netzker R, Aulwurm U, Hermfisse U, Fabian D et al. (2000) Control of thymocyte proliferation via redox-regulated expression of glycolytic genes. Redox Rep $5(1): 52-54$.

[25] Kim JW, Dang CV (2005) Multifaceted roles of glycolytic enzymes. Trends Biochem Sci 30:142-150.

[26] Pastorino JG, Hoek JB, Shulga N (2005) Activation of glycogen synthase kinase 3b disrupts the binding of hexokinase II to mitochondria mitochondria by phosphorylating voltage-dependent anion channel and potentiates chemotherapy-induced cytotoxicity. Cancer Res 65:10545-10554.

[27] Gatenby RA, Gillies RJ (2004) Why do cancers have high aerobic glycolysis? Nat Rev Cancer 4:891-899.

[28] Ganapathy-Kanniappan S, Geschwind JF (2013) Tumor glycolysis as a target for cancer therapy: progress and prospects. Mol Cancer 12(152):4598-12.

[29] Kondoh H, Lleonart ME, Bernard D, Gil J (2007) Protection from oxidative stress by enhanced glycolysis; a possible mechanism of cellular immortalization. Histol Histopathol 22:85-90.

[30] Ganapathy-Kanniappan S, Geschwind JF (2013) Tumor glycolysis as a target for cancer therapy: progress and prospects. Mol Cancer 12(152):4598-12.

[31] Bonnet S, Archer SL, Allalunis-Turner J, Haromy A, Beaulieu C et al. (2007) A mitochondria-K+ channel axis is suppressed in cancer and its normalization promotes apoptosis and inhibits cancer growth. Cancer cell 11(1):37-51.

[32] Santidrian AF, Matsuno-Yagi A, Ritland M, Seo BB, LeBoeuf SE, Gay LJ, et al (2013) Mitochondrial complex I activity and NAD+/NADH balance regulate breast cancer progression. J Clin Invest 123(3):1068-1081.

[33] Schulz TJ, Thierbach R, Voigt A, et al (2006) Induction of oxidative metabolism by mitochondrial frataxin inhibits cancer growth: Otto Warburg revisited. J Biol Chem 281:97781.

[34] Gogvadze V (2011) Targeting mitochondria in fighting cancer. Curr Pharm Design 17(36):4034-4046. 
[35] Lu H, Burns D, Garnier P, Wei G, Zhu K, Ying W (2007) P2X 7 receptors mediate NADH transport across the plasma membranes of astrocytes. Biochem Bioph Res Co 362(4):946-950.

[36] Fisher DJ, Fernández RE, Maurelli AT (2013) Chlamydia trachomatis transports NAD via the Npt1 ATP/ADP translocase. J Bacteriol 195(15):3381-3386.

[37] Pelzmann B, Hallström S, Schaffer P, Lang P, Nadlinger, K, Birkmayer GD, et al (2003) NADH supplementation decreases pinacidil-primed IK (ATP) in ventricular cardiomyocytes by increasing intracellular ATP. Brit J Pharmacol 139(4):749-754.

[38] Rex A, Hentschke MP, Fink H (2002) Bioavailability of Reduced Nicotinamide-adenin-dinucleotide (NADH) in the Central Nervous System of the Anaesthetized Rat Measured by Laser-Induced Fluorescence Spectroscopy. Pharmacol Toxicol 90(4):220-225.

[39] Tse MT (2010) Muscle disorders: Preventing wastage. Nat Rev Drug Discov 9(10):763763.

[40] Leij-Halfwerk S, Agteresch HJ, Sijens PE, Dagnelie PC (2002) Adenosine triphosphate infusion increases liver energy status in advanced lung cancer patients: An in vivo ${ }^{31} \mathrm{P}$ magnetic resonance spectroscopy study. Hepatology 35(2):421-424.

[41] Haskell CM, Wong M, Williams A, Lee LY (1996) Phase I trial of extracellular adenosine 5'-triphosphate in patients with advanced cancer. Med Pediatr Oncol 27:165-73.

[42] Haskell CM, Mendoza E, Pisters KM, Fossella FV, Figlin RA (1998) Phase II study of intravenous adenosine 5'-triphosphate in patients with previously untreated stage IIIB and stage IV non-small cell lung cancer. Invest New Drugs 16:81-5.

[43] Agteresch HJ, Dagnelie PC, van der Gaast A, Stijnen T, Wilson JH (2000) Randomized clinical trial of adenosine 5'-triphosphate in patients with advanced non-small-cell lung cancer. J Natl Cancer Inst 92:321-8.

[44] Jatoi A, Loprinzi CL, Sloan J, Goldberg RM (2000) Is ATP (Adenosine 5'Triphosphate), like STP®, a Performance-Enhancing Additive for the Tanks of Cancer Patients? J Natl Cancer Inst 92(4):290-291.

[45] Agteresch HJ, Rietveld T, Kerkhofs LGM, et al (2002) Beneficial effects of adenosine triphosphate on nutritional status in advanced lung cancer patients: A randomized clinical trial. J Clin Oncol 20:371-378.

[46] Jatoi A, Loprinzi CL (2002) Adenosine triphosphate: Does it help cancer patients "get bigger and stronger"? J Clin Oncol 20(2):362-363.

[47] Beijer S, Gielisse EA, Hupperets PS, van den Borne B, van den Beuken-van Everdingen M, Nijziel MR, et al. (2007) Intravenous ATP infusions can be safely administered in the home setting: a study in pre-terminal cancer patients. Invest New Drug 25(6):571-579.

[48] Chaudry IH (1982) Does ATP cross the cell plasma membrane. Yale J Biol Med 55(1):1. 
[49] Zhai X, Yang Y, Wan J, Zhu R, Wu Y (2013) Inhibition of LDH-A by oxamate induces G2/M arrest, apoptosis and increases radiosensitivity in nasopharyngeal carcinoma cells. Oncol Rep 30(6):2983-2991.

[50] Chevrollier A, Loiseau D, Chabi B, Renier G, Douay O, Malthièry Y, Stepien G (2005) ANT2 isoform required for cancer cell glycolysis. J Bioenerg Biomembr 37(5):307-317.

[51] Le Bras M, Borgne-Sanchez A, Touat Z, El Dein OS, Deniaud A, Maillier E, et al (2006) Chemosensitization by knockdown of adenine nucleotide translocase-2. Cancer Res 66(18):9143-9152.

[52] Gallerne C, Touat Z, Chen ZX, Martel C, Mayola E, Buron N, et al (2010) The fourth isoform of the adenine nucleotide translocator inhibits mitochondrial apoptosis in cancer cells. Int J Biochem Cell B 42(5):623-629.

[53] Jang JY, Choi Y, Jeon YK, Aung KC, Kim CW (2008) Over-expression of adenine nucleotide translocase 1 (ANT1) induces apoptosis and tumor regression in vivo. BMC cancer 8(1):160.

[54] Grover GJ, Malm J (2008) Pharmacological profile of the selective mitochondrial F1F0 ATP hydrolase inhibitor BMS-199264 in myocardial ischemia. Cardiovasc Ther 26:287-296.

[55] Grover GJ, Marone PA, Koetzner L, Seto-Young D (2008) Energetic signalling in the control of mitochondrial F1F0 ATP synthase activity in health and disease. Int J Biochem Cell Biol 40:2698-2701.

[56] Moreno-Sánchez R, Rodríguez-Enríquez S, Saavedra E, Marín-Hernández A, Gallardo-Pérez JC (2009) The bioenergetics of cancer: is glycolysis the main ATP supplier in all tumor cells? Biofactors 35(2): 209-225.

[57] Seyfried BT, Kiebish M, Marsh J, Mukherjee P (2009) Targeting energy metabolism in brain cancer through calorie restriction and the ketogenic diet. J Cancer Res Ther 5(1):S7-15.

[58] Slade NEDA., Storga-Tomic D, Birkmayer GD, Pavelic KRE S IMR, Pavelic JASMINKA (1999) Effect of extracellular NADH on human tumor cell proliferation. Anticancer Res 19(6/B):5355-5360.

[59] Birkmayer GD, Zhang J (2003) NADH in Cancer Prevention and Therapy. Phytopharmaceuticals in Cancer Chemoprevention. CRC series in Modern Nutrition Science, CRC Press, FL, USA.

[60] Birkmayer JGD, Birkmayer W (1991) The co-enzyme nicotinamide adenine dinucleotide (NADH) as biological antidepressant agent. Experience with 205 patients. New Trends Neuropharmacol 5:15-23.

[61] Birkmayer JGD, Vrecko C, Volc D, Birkmayer W (1993) Nicodinamide adenine dinucleotide (NADH) - a new therapeutic approach to Parkinson's disease. Comparison of oral and parenteral application. Acta Neurol Scand 146:32-35. 
[62] Forsyth LM, Preuss HG, Macdowell AL, Chiazze JR, Birkmayer GD, Bellanti JA (1999) Therapeutic effects of oral NADH on the symptoms of patients with chronic fatigue syndrome. Ann. Allergy Asthma Immunol 82:185-191.

[63] Kuhn W, Muller T, Winkel R, Danielczik S, Gerstner A, Hacker R, Mattern C, Przuntek H (1996) Parenteral application of NADH in Parkinson's disease: clinical improvement partially due to stimulation of endogenous levodopa biosynthesis. J Neural Transm 103:1187-1193. 